\title{
INVESTIGACIONES
}

\section{Tensiones en la construcción de identidades académicas en una universidad chilena*}

\author{
Tensions in the construction of academic identities in a Chilean university
}

\author{
Carolina Guzmán-Valenzuela, ${ }^{a}$ María Martínez Larraín ${ }^{b}$ \\ aCentro de Investigación Avanzada en Educación CIAE, Universidad de Chile \\ Telf.: (56) 229781207. Correo electrónico: carolina.guzman@ ciae.uchile.cl \\ bEscuela de Auditoría, Facultad de Ciencias Económicas, Jurídicas y Ciencias Sociales, \\ Universidad de Valparaíso \\ Telf.: (56) 322117464. Correo electrónico: mariateresa.martinez@uv.cl
}

\begin{abstract}
RESUMEN
En este artículo se analizan los modos en que los académicos construyen su identidad en una universidad chilena. Ser un académico conlleva un estatus ontológico (quién es el académico) y las tareas adscritas al rol de ser académico. Este es un estudio cualitativo a través del cual se llevaron a cabo 20 entrevistas con académicos de diversas características y se analizaron documentos institucionales para situar el fenómeno de construcción de identidad académica. Los resultados ponen en tensión las visiones ideales versus las reales en relación a las tareas asociadas al rol académico. Los académicos refieren el condicionamiento de su rol a factores individuales, institucionales y más globales. El análisis permite identificar un interjuego entre macro estructuras y la agencia que ejercen los académicos. Este tipo de estudio contribuye al entendimiento de la profesión académica y a las políticas institucionales que responden a demandas globales y nacionales.
\end{abstract}

Palabras clave: identidad, rol del profesor, universidad, investigación cualitativa, análisis cualitativo.

\section{ABSTRACT}

This paper presents an analysis of the ways in which academics build their identities in a Chilean university. Being an academic involves both an ontological status (who the academic is) and, the tasks attached to the role. This study uses a qualitative approach through which 20 interviews with academics of diverse characteristics were conducted. Also, several institutional documents were analyzed in order to gain a sense of the institutional context. Results show a tension between academics' ideal visions about being an academic and the tasks they have to conduct. Academics also refer to how their identities are conditioned by individual, institutional and global factors. The analysis allows the identification of a confrontation between macro-structures and agentic behaviors on the part of academics. This study might help to understand the academic profession as well as to contribute to both public and institutional policies that respond to global and national tendencies.

Key words: identity, role of the professor, university, qualitative research, qualitative analysis.

\footnotetext{
Agradecimientos a la Comisión Nacional de Ciencia y Tecnología de Chile (CONICYT) a través del FONDECYT de Inicio 11110102. También agradecemos al programa basal PIA-para Centros de Excelencia, Proyecto BF0003.
} 


\section{INTRODUCCIÓN}

Históricamente, ser académico ha involucrado labores de tipo docente, de investigación y gestión y, en algunos casos de 'vinculación con el medio', así como múltiples tareas administrativas y de tipo burocrático (Guzmán-Valenzuela y DiNapoli, 2014; Ylijoki y Mäntylä, 2003). Sin embargo, a diferencia del pasado, los académicos y las universidades a las que pertenecen se encuentran bajo una presión creciente por ser productivos, especialmente en lo concerniente a investigación (Berríos, 2008; Gil-Antón, 2000; McAlpine y Amundsen, 2011). Lo anterior ha llevado a que las tareas de investigación y docencia se vean enfrentadas en la medida que la primera tiende a ser más valorada en términos de prestigio y status (Berríos, 2007; Clegg, 2008; Grediaga, 2000, 2001) y, en ciertos casos (como el de Chile y México), conlleva la posibilidad de ingresos financieros extra y directos. Este estudio se centra en Chile, país con uno de los sistemas de educación superior más mercantilizados del mundo (OECD, 2012) y en donde las presiones en torno a la productividad académica impactan en las formas de ser académico.

La identidad académica resulta relevante de estudiar por varias razones. Por un lado, tal como se ha dicho, la identidad académica conlleva una serie de roles y tareas asociadas que se superponen y chocan entre sí y que se ven afectadas por estructuras nacionales y globales que lo vuelven un constructo cambiante y difuso, con múltiples definiciones, y que obedece también a contextos institucionales particulares. Por otro lado, si bien el estudio de la profesión académica ha sido desarrollado ampliamente en países anglosajones (Barnett y DiNapoli, 2008; Clegg, 2008; Fanghanel, 2012; Henkel, 2005), en Iberoamérica los estudios son más bien limitados, para detalles ver estudios iniciados a fines de los años 90 y principios del 2000 en México (Galaz 1999; Gil-Antón, 2000; Grediaga, 2000, 2001, 2007; Salord, 2001) y en Chile (Berríos, 2007, 2008; Guzmán-Valenzuela y Barnett, 2013a, 2013b; Lolas, 2008).

Las preguntas de investigación discutidas en este artículo son: ¿cómo definen los académicos 'ser académico'? ¿Qué roles conlleva la profesión académica? ¿Cómo manejan, complementan y priorizan los académicos sus diversos roles y tareas atendiendo sus propios proyectos académicos y los de la universidad a la que pertenecen?

\section{LA CONSTRUCCIÓN DE LA IDENTIDAD ACADÉMICA}

La naturaleza de la identidad puede interpretarse como proceso y producto de un juego de negociaciones de significados y de toma de posiciones en las que intervienen diferentes tipos de entidades, experiencias y significados construidos socialmente. Involucra un interjuego entre las estructuras y los agentes (Archer, 1995, 2003), entendidos estos últimos de manera individual o colectiva (Fanghanel, 2012) bajo una condición dialógica que tiene lugar de manera permanente a lo largo de la vida (Taylor, 1991 Cit. en Valimäa, 1998).

Entre los elementos conceptuales a precisar en relación al constructo de identidad se encuentra el de rol, que involucra una definición de doble condición en tanto alude a 'quién se es' (identidad) y a 'qué se hace' (actividades/tareas) (Sánchez, 2002). Con respecto al 'ser', el académico se define a sí mismo en un contexto determinado y en el que participa e interacciona con otros; ello supone indagar quiénes son los otros significativos que a partir del diálogo permiten ir construyendo la identidad y cuáles son los grupos de referencia. El 
segundo elemento, es decir 'hacer', se refiere al conjunto de actividades involucradas (tareas académicas), con su propio ordenamiento en un contexto organizativo determinado (Clark, 1991) y que supone tener como marco de referencia las condiciones organizativas, políticas e ideológicas que dan cuenta de estructuras particulares (Archer, 1995, 2003). Fanghanel (2012) señala que el rol académico puede ser explorado analizando las prescripciones para la práctica tanto desde las estructuras como desde los académicos en su rol de agentes. Los académicos, "navegando entre ciertas tensiones" (Fanghanel, 2012, p. 2) que tienen lugar entre la estructura, las comunidades de práctica y ellos mismos, hacen suyos los roles propios del mundo académico. Aquí cobran especial relevancia los grupos particulares y disciplinas (Becher, 2001; Henkel, 2005) a los que pertenecen los académicos - lo que Becher (2001) denomina 'tribus académicas' - en la medida que estos grupos "están íntimamente relacionados con las tareas intelectuales que desempeñan" (Becher, 2001, p. 16) así como con las microprácticas en las que participan los académicos y les hacen estar en el mundo de la universidad (Becher, 2001). Este estudio analiza la construcción de identidades académicas en su doble dimensión de 'ser académico' y las actividades que se realizan ‘como académico’ poniendo especial atención a los conflictos y tensiones experimentadas.

\section{SER ACADÉMICO EN UNA UNIVERSIDAD CHILENA DEFINIDA COMO TRADICIONAL Y COMPLEJA}

Chile cuenta con un sistema de educación superior diversificado, conformado por universidades (estatales y privadas), institutos profesionales y centros de formación técnica. Este estudio se centrará en la perspectiva de académicos que pertenecen al primer tipo de instituciones. Actualmente, dentro de las universidades chilenas existe un grupo compuesto por las denominadas universidades tradicionales (Brunner, 1997) —que incluye 16 universidades estatales y 9 privadas-, y universidades no tradicionales que son privadas (alrededor de 40). Son las universidades tradicionales las que históricamente han obtenido mayor financiamiento para realizar investigación a través del Fondo Nacional de Desarrollo Científico y Tecnológico (FONDECYT). Lo anterior lleva a hacer una distinción entre universidades que realizan investigación y docencia (generalmente las universidades tradicionales) versus las centradas exclusivamente en docencia (Bernasconi, 2006). Otra distinción la lleva a cabo Merino (2004), quien diferencia universidades 'complejas' de las 'no complejas', de manera que las primeras llevan a cabo múltiples funciones como docencia, investigación, creación artística y servicio a la sociedad; en cambio las universidades no complejas están centradas en la función docente.

Las distinciones hechas guardan relevancia en la medida en que las maneras de definir una universidad impactan en las formas de construir la identidad académica y ser académico. Guzmán-Valenzuela y Barnett (2013a) analizan cómo una universidad chilena orientada a la docencia deja menos espacio a los académicos para llevar a cabo tareas relacionadas con la investigación; en cambio, una universidad definida como compleja abre un campo de posibilidades que deja mayor espacio para la diversidad de roles y tareas, aunque también imprime mayor complejidad al ejercicio de rol. Con el objetivo de rescatar las significaciones en torno a la construcción de identidades académicas y la diversidad de roles y tareas asociadas, este estudio fue llevado a cabo en una universidad chilena estatal y tradicional considerada compleja. 


\section{MÉTODO}

Esta investigación se adscribe a un paradigma interpretativo (Denzin y Lincoln, 2008), pues busca comprender las significaciones que un grupo de académicos chilenos otorga a la experiencia de ser académicos universitarios. Estas asignaciones de significados son dinámicas, pudiendo ser estos últimos construidos, transformados e intencionalmente reconstruidos en negociaciones permanentes con otros en un contexto singular de acción humana (Erickson, 1989). Es importante señalar aquí que dichas significaciones son subjetivas, solamente pueden ser comprendidas en el contexto particular del que forman parte y no pretenden ser representativas ni extrapolables a otras realidades, aunque sí pueden constituirse en un aporte para iniciar investigaciones similares en otros contextos.

Como estrategia de investigación se utilizó de estudio de caso (Merriam, 1998) que consiste en el estudio profundo de la particularidad, complejidad y de los procesos interactivos de un fenómeno, en este caso la construcción de las identidades de académicos universitarios en un contexto particular: una universidad chilena considerada como compleja (Merino, 2004). Esta universidad es estatal y está compuesta por 10 Facultades y 2 Campus. A la fecha del estudio contaba con una matrícula aproximada de 16000 estudiantes en carreras profesionales, 746 en programas de postgrado, 320 cursando especialidades profesionales. Sus áreas de función declaradas corresponden a docencia (profesional y licenciatura, pre y postgrado, postítulo), investigación y extensión.

Los participantes de este estudio corresponden a 20 académicos cuya selección fue efectuada en base a criterios (Goetz y LeCompte, 1988), teniendo en cuenta la variación de características tales como género, disciplina, años de trayectoria académica y tipo de jornada laboral (jornada completa/jornada parcial), entre otros (ver Tabla 1).

Tabla 1. Características de los participantes del estudio

\begin{tabular}{|c|c|c|c|c|c|c|}
\hline $\mathrm{N}^{\circ}$ & $\begin{array}{c}\text { Género } \\
\text { (Femenino/ } \\
\text { Masculino) }\end{array}$ & $\begin{array}{c}\text { Tipo de jornada } \\
\text { (Tiempo completo: } \\
\text { TC; Tiempo } \\
\text { parcial: TP) }\end{array}$ & $\begin{array}{c}\text { Años de } \\
\text { experiencia } \\
\text { como } \\
\text { académico }\end{array}$ & Disciplina & $\begin{array}{c}\text { Estudios de } \\
\text { post-grado } \\
(\mathrm{PhD} \text { o } \\
\text { Magíster }(\mathrm{Mg})\end{array}$ & $\begin{array}{c}\text { Cargo gestión/ } \\
\text { directivo }\end{array}$ \\
\hline 1 & $\mathrm{~F}$ & $\mathrm{TC}$ & 12 & Psicología & Sí (PhD) & No \\
\hline 2 & M & $\mathrm{TC}$ & 8 & Obstetricia & Sí (PhD) & Sí \\
\hline 3 & M & $\mathrm{TC}$ & 17 & Diseño & Sí (PhD) & No \\
\hline 4 & M & $\mathrm{TC}$ & 18 & Administración & Sí (Mg) & Sí \\
\hline 5 & $\mathrm{~F}$ & $\mathrm{TC}$ & 17 & Obstetricia & Sí (Mg) & No \\
\hline 6 & $\mathrm{~F}$ & $\mathrm{TC}$ & 1,5 & Auditoría & No & No \\
\hline 7 & $\mathrm{~F}$ & $\mathrm{TP}$ & 4 & Biología & Sí (Mg) & No \\
\hline 8 & M & $\mathrm{TP}$ & 37 & Ingeniería & Sí (Mg) & No \\
\hline 9 & $\mathrm{~F}$ & $\mathrm{TP}$ & 3 & Biología & Sí (Mg) & No \\
\hline
\end{tabular}




\begin{tabular}{|c|c|c|c|c|c|c|}
\hline 10 & F & TP & 25 & Ingeniería & No & No \\
\hline 11 & M & TP & 10 & Ingeniería & Sí (Mg) & No \\
\hline 12 & M & TP & 4 & Derecho & Sí (PhD) & Sí \\
\hline 13 & M & TC & 42 & Biología & Sí (Mg) & Sí \\
\hline 14 & M & TP & 17 & Ingeniería & Sí (Mg) & No \\
\hline 16 & F & TP & 26 & $\begin{array}{c}\text { Química/ } \\
\text { farmacia }\end{array}$ & Sí (Mg) & Sí \\
\hline 17 & F & TC & 21 & $\begin{array}{c}\text { Trabajo social } \\
\text { Construcción }\end{array}$ & Sí (Mg) $(\mathrm{Mg})$ & Sí Sí \\
\hline 18 & M & TP & 9 & $\begin{array}{c}\text { Profesor de } \\
\text { Historia y } \\
\text { Geografía }\end{array}$ & No & Sí \\
\hline 19 & M & TP & 35 & Odontólogo & No & No \\
\hline 20 & M & TP & 9 & Psicología & No & No \\
\hline
\end{tabular}

Fuente: Elaboración propia

En total se llevaron a cabo 20 entrevistas semiestructuradas a académicos. Todos ellos firmaron consentimientos informados antes de la producción de datos de acuerdo a estándares éticos establecidos por los comités de ética nacionales. Su participación fue libre y voluntaria y se garantizó mantener el anonimato y confidencialidad de los datos. Las entrevistas tuvieron una duración de 60 a 75 minutos, en un lapso de 8 meses entre los años 2011-2013, en las cuales se abordaron las siguientes cuestiones: el quehacer cotidiano y los significados personales asociados a ser académico; percepciones acerca de sus roles y tareas que desempeñan; intereses académicos; formas de organización y priorización de sus actividades académicas; facilitadores y obstaculizadores en su labor académica. Todas las entrevistas fueron registradas por medio de audio digital, transcritas, revisadas y cada uno de los entrevistados pudo leer y plantear su conformidad con el rigor del proceso señalado para su posterior análisis, lo cual constituye un criterio de calidad para investigaciones cualitativas (Guba, 1989) denominado control de los miembros (Vasilachis, 2006). Antes de iniciar la entrevista, a cada académico, se le aplicó una pequeña encuesta para recabar información sobre el tipo de jornada laboral, grado académico, principales tareas desempeñadas y experiencia académica. Dicha información fue sistematizada y revisada y permitió ir seleccionando los siguientes participantes de manera de lograr variabilidad en las características de los mismos.

Asimismo, se llevó a cabo un análisis documental que consideró los principales documentos institucionales de la universidad estudiada (proyecto educativo, informe de evaluación institucional y páginas web de la universidad) logrando así una mejor comprensión del contexto de estudio. El uso de diversas técnicas de producción de datos 
(entrevistas más análisis documental) contribuyó a la triangulación de las fuentes de información (Guba, 1989) como criterio de calidad (Vasilachis, 2006).

Los datos fueron analizados a través de la técnica de análisis de contenido. Tres miembros del equipo trabajaron primero por separado y después en conjunto — durante 12 reuniones de equipo de una duración aproximada tres horas cronológicas - en la categorización de la información, ayudados por el software Atlas-ti. En dichos encuentros se establecieron y consensuaron progresivamente los códigos descriptivos de bajo nivel inferencial o inductivos, así como los códigos deductivos (provenientes de la teoría), inspirados principalmente en autores como Archer (1995, 2003), Becher (2001), Barnett (2011) y Fanghanel (2012). Con lo anterior se logró la triangulación de perspectivas de investigadores (Patton, 1999), lo que constituye otro criterio de calidad sugerido por Guba (1989). El análisis arrojó un total de 679 códigos de distinto nivel de abstracción, es decir, códigos inductivos y códigos deductivos. Dichos códigos se agruparon y se establecieron dimensiones de análisis o categorías de mayor nivel de abstracción (Van Maanen, 1982) que respondían a las preguntas de investigación del estudio. En la Tabla 2 se presenta un ejemplo con dos dimensiones de análisis junto con su definición y algunos códigos que las componen.

Tabla 2. Ejemplo de dimensión de análisis, definición y códigos asociados

\begin{tabular}{|l|l|l|}
\hline Dimensión de análisis & \multicolumn{1}{|c|}{ Definición del código } & \multicolumn{1}{|c|}{$\begin{array}{c}\text { Ejemplos de códigos inductivos (CI) } \\
\text { y código deductivos (CD) }\end{array}$} \\
\hline Función docente & $\begin{array}{l}\text { Se refiere a las tareas de } \\
\text { docencia desempeñadas } \\
\text { por los académicos y a } \\
\text { las percepciones } \\
\text { asociadas }\end{array}$ & $\begin{array}{l}\text { CI: Ejerio de docencia como tarea } \\
\text { prescriptiva } \\
\text { CI: Ejercicio de la docencia como tarea } \\
\text { irrenunciable } \\
\text { CI: Ejercicio de la docencia como tarea que } \\
\text { gusta y motiva pero que desgasta } \\
\text { CI: La enseñanza como una tarea que quita } \\
\text { tiempo para la investigación }\end{array}$ \\
\hline Tareas de investigación & $\begin{array}{l}\text { Se refiere a las tareas } \\
\text { investigativas } \\
\text { desempeñadas por los } \\
\text { académicos y a las } \\
\text { percepciones asociadas }\end{array}$ & $\begin{array}{l}\text { CDealización de investigación como parte } \\
\text { fundamental del quehacer (asociado a la } \\
\text { disciplina) } \\
\text { CI: Realización de investigación como } \\
\text { iniciativa personal } \\
\text { CD: Motivación y gusto por la investigación }\end{array}$ \\
\hline
\end{tabular}

Fuente: Elaboración propia

Para efectos de este artículo presentamos los resultados organizados de acuerdo a dos preguntas de investigación: significaciones acerca de qué es ser académico y significaciones referidas a las tareas desempeñadas por los académicos (docencia, investigación, gestión, extensión y administración). Cada una de estas dimensiones son ilustradas con fragmentos provenientes de las entrevistas, para lo cual se utilizan las siglas 'E' (entrevista) y el número correspondiente de entrevista (ver Tabla 1). 


\section{RESULTADOS}

\subsection{QUÉ SIGNIFICA SER ACADÉMICO}

Los significados sobre ser académico son expresados en primer lugar desde un deber ser: en una universidad compleja como la aquí estudiada, ser académico significa atender a múltiples tareas, haciéndose necesaria la distinción entre ser un académico y ser o un docente o un investigador.

"Un académico es... un profesional, el cual trabaja principalmente en... universidades, desempeñando... una serie de funciones: docencia... pregrado y postgrado, investigación, extensión y administración y/o gestión. O sea, un académico debería hacer todo eso a mi entender, para distinguir quizás un poco de aquellos colegas que solo desarrollan docencia; o sea, ese es un profesor, y de aquellos colegas que solo hacen investigación - que esos son investigadores- por eso un académico es más amplio" (E1).

Para los entrevistados la profesión académica se estructura a partir de diferentes actividades descritas como complementarias, necesarias e importantes para el desarrollo de una universidad (E2, E4, E6, E7, E9, E10, E12, E13, E15, E18). Todas ellas guardan relación con el conocimiento, ya sea con su creación, transmisión o transferencia, percibiéndose como elemental la actitud del rigor, la excelencia y la actualización permanente.

Por otro lado, desde las perspectivas que atienden al quehacer cotidiano en la universidad y sus condiciones de ejercicio, se reconoce la necesidad de una focalización en ciertos roles. La mayoría de los académicos afirma dedicarse solo a algunos tipos de actividad o función universitaria, aun cuando aseguran la importancia del ser académico desde el ejercicio de múltiples roles. Se originaría una especie de división del trabajo que lleva a una diferenciación de los académicos de acuerdo con los principales roles que ejercen. ¿En base a qué criterios se dedican más a unas que a otras tareas? El análisis de las entrevistas permite distinguir entre preferencias y factibilidad, tal y como se describe a continuación:

- Circunscripción por preferencia. La circunscripción a unas determinadas tareas estaría dada por el deseo, afinidad e inclinación hacia una tarea, o bien en virtud del despliegue de una habilidad particular (E4, E6, E7, E10, E11, E16), dando cuenta de criterios subjetivos que juegan un papel en las decisiones de los académicos, aun estando situados en un contexto institucional que plantea prioridades.

Las personas también tenemos prioridades,... en el caso académico yo percibo que, para mí situación personal, las prioridades tienen que ver fuertemente con lo que yo quiero o no quiero hacer, con lo que me gusta o no me gusta hacer. (E4).

- $\quad$ Circunscripción por motivos de factibilidad y limitaciones de tiempo. Se responsabiliza a las condiciones y/o limitaciones de carácter estructural, como la misión de la carrera/ facultad (más centrada en crear y/o aplicar nuevos conocimientos y/o hacer docencia, por ejemplo), las disciplinas cultivadas y la asignación de cargos y de recursos disponibles, horas de contrato, tal y como queda reflejado en el siguiente fragmento.

La facultad exige ciertos proyectos, por ejemplo, la creación de magíster, la creación de diplomados, planes de mejoras, la construcción de un plan estratégico y también está el tema de la acreditación de la carrera. Y yo tengo ciertas horas asignadas para apoyar al secretario académico que es el gestor de todo ese tipo de proyectos. (E6). 
No obstante lo anterior, hay académicos (E2, E3, E4, E8, E9, E12, E13, E16) que expresan sus intentos cotidianos por cubrir y compatibilizar los diferentes roles que forman parte del ideal de ser académico.

Tengo que estar dividiendo mi horario en que los alumnos me llaman, que el laboratorio... - como te decía - que el pago de las facturas, que el programa. Y, además, tengo una clase entremedio..., estoy transcribiendo un paper, tengo que mandarlo y... ahora este año me invitaron a ser miembro de una de las agencias de acreditación para carreras de la salud, como par evaluador me tocaron dos procesos de acreditación (E2).

\subsection{ROLES Y TAREAS DEL ACADÉMICO}

Más allá de su dedicación, ya sea múltiple o focalizada ¿Cómo los académicos van significando el ejercicio de sus múltiples roles y tareas? A continuación se presenta un análisis descriptivo más detallado en torno a las tareas de docencia, investigación y gestión que expresan desarrollar los académicos de esta universidad.

\subsubsection{Docencia}

La docencia es una tarea esencial para los académicos entrevistados (ver Tabla 1). De acuerdo a los documentos institucionales, es concebida como labor primordial, de manera tal que todo académico debe destinar — aunque sea en un porcentaje mínimohoras de docencia en su carga académica. Según los académicos, la docencia es uno de los principales ejes que define la identidad académica y una razón de ser en su labor académica en esta universidad (E1, E3, E5, E6, E7, E9, E10, E11, E12, E14, E15, E20).

... yo creo que hay que hacer un mínimo de docencia... yo creo que no hay que permitir que haya docentes o académicos sin docencia, me parece que esto no es un centro de investigación, esto es una universidad. (E12).

La docencia supone para los académicos un trabajo indirecto que requiere tiempo adicional para su adecuado desarrollo. Incluye tareas de planificación de clases, preparación de material de enseñanza y evaluación de los aprendizajes, dedicación a la atención de estudiantes fuera de clase, e incluso el perfeccionamiento por cuenta propia cuando se ha percibido como necesario (E1, E3, E4, E5, E6, E7, E9, E10, E11, E14, E17, E18, E19). En el caso de profesores a honorarios, tales horas indirectas son inexistentes, y en el caso de profesores con contrato se contemplan horas ('indirectas') para llevar a cabo estas tareas. Los académicos entrevistados consideran esta labor importante, aunque no reconocida, señalando que deben echar mano a sus tiempos personales para realizarlas de manera satisfactoria.

Se evidencia así una precarización del trabajo docente y sus condiciones de realización para ambas realidades laborales, implicando un desgaste y cansancio que para los académicos entrevistados no es reconocido por la institución.

Asimismo existen otras particularidades en esta universidad, relativas a los tipos de jornada de los académicos que contribuyen a la precarización de la docencia. Para el caso de académicos con jornadas parciales o que prestan servicios por horas, "ser académico" se 
circunscribe exclusivamente a la función docente, en oposición al académico que cumple diversos roles (y que suele tener jornada completa). Se aprecia la expresión del llamado profesor taxi (Simbürger, 2013), cuyas condiciones laborales se describen como inestables y de sobredemanda. Ante dicha fragilidad, se sostiene que el docente queda sujeto (ante necesidades financieras) a asumir una carga académica (número de cursos a impartir), dudando de la calidad de la enseñanza impartida.

Hoy día las remuneraciones de los académicos... se han ido estrechando y eso obliga a que los académicos tengan que correr de un lado para otro. Por ahí alguien decía: 'nos hemos convertido en taxi teachers'... Tú entenderás que a razón de cien, ciento cincuenta mil pesos mensuales por cátedra, para que tú te puedas hacer un sueldo, una remuneración, no sé, pongámosle un millón y medio, tendrías que dictar diez cátedras...Y si quieres optar a algo un poquitito mayor, tendrías que estar haciendo quince a veinte cátedras, o sea, ¿qué calidad académica logras con eso? (E14).

Esta noción, criticada por varios académicos, sobre la docencia bajo una óptica de mercado y rentabilidad contradice las cualidades enunciadas para el "Ser académico", y contribuiría a la desvalorización del rol docente en tanto este no hace una docencia guiada por criterios de excelencia, sino que por criterios mercantiles.

\subsubsection{Investigación}

Si bien la labor docente es significada como una actividad fundamental del ser académico, en gran parte de los casos la investigación también es concebida como una tarea académica esencial (E1, E2, E4, E8, E7, E9, E12, E13, E15, E16, E18). Al respecto corresponde precisar el caso de académicos que sitúan la investigación como su actividad principal o de base en su quehacer (E1, E2, E7, E9, E12, E13, E16), la cual es calificada como necesaria y gratificante a pesar del nivel de demanda y responsabilidad asociadas. De este modo la investigación se configura como una actividad preferida para algunos académicos, tal y como se lee en la siguiente cita:

Yo creo que mi sustento académico es la investigación. Yo renunciaría a todo para ir a una investigación... O sea, si me dijeran que no vaya, no vaya a hacer clases... 'Okey'. No me dolería. Pero si a mí me dijeran: 'tienes que dejar de hacer investigación, para dedicarte a la docencia'... yo creo que no lo podría soportar. (E16).

Otros académicos visualizan la docencia y la investigación como tareas enfrentadas en términos de grados de responsabilidad o de reconocimiento por parte de los colegas y la misma institución. Finalmente, se deben reconocer los casos en que por condiciones institucionales, la investigación ha debido ser abandonada o postergada (por ejemplo al ocupar cargos de gestión) a pesar de ser una tarea preferida (E5, E19). En otros casos, la investigación es una actividad deseada pero incipientemente desarrollada o no iniciada por restricciones institucionales, ya que el tiempo debe ser dedicado a la gestión o a la docencia (E6, E14, E17).

Ha sido un..., un recorrido... de querer llegar siempre a una meta y, y encontrar varias barreras... sobre todo en el área de la investigación. Cero barreras para hacer clases, me 
llovían las propuestas para hacer clases y las tomé todas; cero barreras para hacer extensión —nadie las hacía-, tomé lo que nadie hacía y lo armé.... Cero barreras también con el tiempo... para hacer actividades de gestión académica,.... pero muchas barreras para la investigación..., era como una suerte de terreno prohibido... (E5).

Por otro lado, la actividad investigativa — entendida como investigación financiada con fondos externos - genera sentimientos encontrados entre los académicos. Por un lado, se considera que otorga relevancia y prestigio a una universidad y sus académicos, por lo que debiera tener mayor reconocimiento y ser más incentivada (E7, E11, E1). Pero, al mismo tiempo, se la concibe como una actividad utilitaria que puede cumplir varios fines: mejorar indicadores de acreditación institucional (E1, E7), mejorar los ingresos salariales, o bien como una práctica competitiva que involucra postular a fondos limitados que fomentan el individualismo e impactan en la forma de llevar a cabo el trabajo académico.

Cuando volví [del doctorado] logré ubicarme en proyectos de investigación importantes. He tenido y/o he estado en proyectos FONDECYT y FONDEF financiados por CONICYT... Me ha permitido hacer cosas que de otra forma hubiera sido muy difícil realizar. Además, eso me ha dado mayor tranquilidad económica porque dichos fondos permiten el pago de incentivos. El problema de cómo están concebidos los fondos es que son demasiados competitivos a nivel individual y eso genera individualismo que se puede transformar en canibalismo. (E8).

Asimismo, hay tensiones con respecto a la inversión de tiempo y dedicación que requiere la función investigativa. La compleja naturaleza de esta labor involucra desde la generación de proyectos y su administración y desarrollo hasta la comunicación de los resultados (a través de conferencias, encuentros científicos o congresos, la escritura de libros y artículos). Particularmente, la publicación en revistas indexadas reconocidas se transforma en un elemento crucial de legitimación de las actividades de investigación, especialmente para el académico joven.

Hay académicos que consideran que el mayor beneficio de este trabajo se lo lleva la universidad y ellos deben enfrentar barreras como poca valoración de la actividad y una serie de trabas burocráticas. No obstante, a pesar de estas dificultades, fue posible observar el sentimiento que predomina en relación a la gratificación por el ejercicio de este rol. Algunos académicos subrayan el desarrollo del potencial creativo del académico (E12) y de los desafíos que enfrentan cuyos resultados son reconfortantes. Otros, relatan sus experiencias investigativas poniendo énfasis en iniciativas en las que tienden a producir rupturas (E9, E13, E16) con sus propias disciplinas, como el caso de un ingeniero que se vincula con la microbiología generando proyectos de investigación interdisciplinares innovadores, o el de una bióloga que se vincula al área del diseño para participar de un proyecto de conservación.

Era un área que no desarrolla la escuela, en general aquí te encuentras con... ingenieros, con constructores que se dedican más a los hormigones, a la gestión, pero estudiar la madera, no hay muchos... eso fue un poco la motivación. Posteriormente, se dieron otras posibilidades... justamente con el estudio de la madera... y eso derivó ya en el estudio de la biología molecular y celular, que es lo que estamos desarrollando ahora... ese es un poquito mi origen, el querer hacer investigación y, y hacer un aporte a mi zona... y fortalecer un área que nunca la escuela la tuvo. (E16). 


\subsubsection{Gestión}

Este estudio consideró la participación de académicos que han asumido responsabilidades de gestión de nivel jerárquico superior dentro de la institución (ya sea en una unidad académica o en el nivel central) aunque también recogió las significaciones de los académicos que no se dedican a la gestión, pero que tienen una clara imagen en torno a esta labor.

A diferencia de lo que ocurre con las actividades de docencia e investigación, la gestión, si bien es descrita como una actividad necesaria en la universidad, parece ser una función de naturaleza no-académica o que implica asumir otra forma de percepción del mundo universitario (E1, E10). Para ejemplificar lo anterior, nótese en el siguiente fragmento la frase ‘cuando vuelva a ser académico' (una vez que se deje el cargo de gestión):

Me interesaba el cargo. Si quiero saber cómo es estar al otro lado y ver la otra cara de la moneda... y a lo mejor voy a cambiar mi punto de vista después, cuando vuelva a ser académico. (E15).

Entre las tareas vinculadas a la gestión se encuentran la formulación, postulación y gestión de proyectos, tareas administrativas (firmas, completación de formularios, gestión de pagos), la participación en reuniones, la gestión de redes, gestión de prácticas, coordinación de unidades, etc., acciones que son vistas como consumidoras de tiempo y que demandan alta responsabilidad así como capacidad de organización adicional por parte del académico (E1, E2, E4, E6, E7, E12, E13, E15, E16). En general, estos tiempos y acciones poseen un mayor grado de dependencia de la gestión de otras personas o unidades lo que conlleva esfuerzo y desgaste y requieren de algún conocimiento específico o experiencia para su realización. Además, para algunos académicos, asumir labores de gestión ha implicado restar tiempo y dedicación en alguna de sus principales y favoritas actividades (E12, E13, E15). Lo anterior permitiría comprender las razones presentadas por los académicos cuando señalan que la gestión 'no es su fuerte' o su rechazo a este tipo de actividades.

Creo que la administración es tiempo perdido... en términos de la gestión académica... es lo que me parece que es menos interesante, menos gratificante y donde uno pierde mucho tiempo. (E12).

Otra perspectiva sobre la actividad de gestión (E2, E4, E8, E13, E15, E20) es aquella que la asume como parte de la madurez del académico, propio de la experiencia. Pudiera ser que estas ideas provienen de la experiencia de ocupar un cargo de gestión, lo que otorga la oportunidad de mirar la labor académica desde una óptica diferente (ya no solamente desde la docencia y la investigación), generando así una visión más comprensiva, estratégica, procesual de la realidad universitaria (E15). Esta perspectiva podría, en cierta forma, representar el discurso oficial dentro de la universidad, justamente por estar asociada a cargos de alta responsabilidad.

Cuando llegué a hacerme cargo de esta dirección, yo también venía sin saber a lo que me tenía que enfrentar acá... ahora, ya desde acá, ya tengo una visión mucho más amplia de la universidad, y eso lo agradezco mucho, ahora conozco... más el quehacer..., aprendo... (E15). 
Este punto de vista sobre la gestión y el ser académico tiende a evidenciar la valoración del quehacer del académico capaz de dar cuenta de una realidad universitaria que requiere ser vista 'desde arriba' e interpretada a la luz de su devenir, sus procesos históricos y su relación con el entorno. La posibilidad de mirar en términos panorámicos la universidad y la vida académica es presentada por académicos dedicados a la gestión, como una necesidad para el desarrollo académico, ya que les permite vincularse con procesos de reflexión que permiten avanzar comunitariamente.

\section{DISCUSIÓN}

En concordancia con lo planteado por Clark (1991) y Bernasconi (2010), para los entrevistados la profesión académica involucra múltiples actividades descritas como complementarias, necesarias e importantes para el desarrollo de cualquier universidad. El conocimiento es eje central del accionar (Clark, 1991) y el académico está llamado a trabajar con este ejerciendo diferentes funciones a nivel de producción, desarrollo y/o comunicación (Becher, 2001). Al mismo tiempo, los académicos dejan entrever que si ellos no llevaran a cabo alguna de estas actividades no podrían ser considerados "académicos". A partir de aquí cabe preguntarse ¿cómo es este proceso de definición de roles y tareas y quién define/decide los cursos de acción de los académicos?

Al analizar los discursos de los académicos en torno a cómo ejercen su rol y las implicancias que esto tiene en la práctica, es posible identificar diversas tensiones. La primera -y más evidente - es una tensión de rol (Fanghanel, 2012; Guzmán-Valenzuela y Barnett, 2013a, 2013b; Lolas, 2008) que da cuenta de una superposición de las tareas de docencia, investigación, gestión y extensión que son altamente demandantes y desgastantes. Estas tensiones conllevan un conflicto (por ejemplo, entre las tareas de docencia e investigación; entre las de investigación y las administrativas o entre las de docencia e investigación y las de gestión y/o administración) que se deja entrever al momento que los académicos deciden qué acciones realizar, focalizan su atención y distribuyen los tiempos en su vida académica. Las decisiones sobre cursos de acción que finalmente llevan a cabo los académicos tienen su origen en una tensión más profunda que traspasa el nivel individual y que da cuenta de un interjuego entre los académicos como agentes y las estructuras de tipo institucional, nacional y global a las que pertenecen (Archer, 1995, 2003). Por un lado, existe el deseo del académico de desenvolverse con cierta autonomía y desarrollar sus proyectos académicos; y por otro, un escenario estructural restrictivo que dictamina qué debe hacer el académico, ya sea desde una lógica más institucional/nacional —pertenencia a una universidad 'compleja' (Merino, 2004) — o global, en la que se pone el acento en la polifunción, la productividad, el control de cuentas y el capitalismo académico (Slaughter y Leslie, 1997).

También existen otras condicionantes estructurales importantes de considerar. Tal y como muestra la literatura, una de ellas guarda relación con el campo disciplinar al que se pertenece (Becher, 2001; Henkel, 2005). Los académicos participantes reconocen que en ciertas facultades o departamentos en los que se privilegia la actividad profesional y la docencia, la investigación queda relegada. Asimismo, la precariedad salarial (GilAntón, 2000) de los académicos conforman un condicionante esencial para llevar a cabo tareas de docencia e investigación, de manera tal que algunos deciden realizar gran 
cantidad de cursos para mejorar el salario y otros optan por recibir incentivos a partir de la investigación con fondos externos competitivos. Esto último genera una particular tensión entre los académicos, quienes distinguen dos tipos de status en la universidad: uno de menor prestigio, generalmente asociado a la docencia, y uno de mayor prestigio asociado a la investigación.

La investigación y la productividad científica como condicionantes estructurales resultan claves para comprender el proceso de construcción de identidades académicas. Por un lado, a nivel individual o grupal se genera la demanda y la presión por mantener el status investigativo creando una 'fragilidad reputacional' (Guzmán-Valenzuela y Barnett, 2013a). Para ello el académico utiliza estrategias tales como la asociación con colegas de otras universidades y disciplinas de manera de conformar una red de investigación que es colaborativa, pero a la vez competitiva (por los recursos) (Becher, 2001). Lo anterior abre la discusión en torno a la competencia en la búsqueda del prestigio y el posicionamiento bajo contextos de precariedad en el acceso a las oportunidades. Situaciones como estas son experimentadas por algunos académicos como un atentando a la comunidad académica, en el sentido de potenciar el comportamiento individualista, competitivo y de exclusión. Por otro lado, a nivel nacional y global la productividad científica se convierte en un objetivo estratégico que obedece a políticas nacionales particulares que responden a las formas en que la universidad del mundo occidental se ha ido configurando y se relaciona con la llamada 'universidad emprendedora' (Clark, 1998).

Pero los cursos de acción de los académicos también dependen de sus intereses y proyectos al interior de ciertas estructuras (Archer, 2003). En una universidad como la estudiada, definida como compleja, existe una diversificación del trabajo (Bernasconi, 2010; Clark, 1991) a partir de la cual el académico intenta desarrollar todas ellas o bien prioriza una sobre otra a partir de sus propios intereses o preferencias. En esta universidad, por ejemplo, el sentimiento de gratificación de rol aparece tanto cuando los académicos están más abocados a la docencia, a la investigación e incluso a la gestión (cuando el académico considera trascendente su trabajo para el logro de objetivos institucionales estratégicos). Como consecuencia, el académico dedica mayor tiempo y energía a alguna(s) de estas tareas por sobre otras, cuestión que ya ha sido explorada y desarrollada en otro artículo (Guzmán-Valenzuela y Barnett, 2013b). Estas gratificaciones parecen ser motores de búsqueda, de adaptaciones y de movimientos a nivel de microprácticas a partir de los cuales los académicos logran sus proyectos y se auto realizan.

\section{CONCLUSIÓN}

A la hora de comprender la construcción de identidades académicas se hace necesario un análisis a nivel individual e institucional, así como nacional (Merino, 2004; Santelices, 2011) y global (Marginson, 2010). Siguiendo los planteamientos de Archer (1993, 2005) es posible distinguir una restricción o condicionamiento por parte de las estructuras (a nivel micro y macro) y a la vez un espacio para acciones disponibles en el quehacer académico (Guzmán-Valenzuela y Barnett, 2013a, 2013b) y que permiten que el académico tome ciertos cursos de acción. Ambos elementos entran en un interjuego constante logrando un cierto equilibrio en el trabajo académico. Una interpretación posible alude a un 'pacto implícito' entre las partes (institución y académicos) de manera que si bien algunas 
prácticas académicas permiten cumplir ciertas exigencias o rutinas universitarias, al mismo tiempo, los académicos se desenvuelven con cierta libertad y actúan en pequeños espacios de práctica según sus intereses y propósitos. Lo anterior se vería facilitado en tanto el sistema universitario se constituye como un sistema en el que sus elementos se encuentran débilmente acoplados permitiendo a algunas partes seguir funcionando con cierta independencia para asumir las demandas conflictivas, responder a requerimientos del medio y adaptarse (Aldrich, 1979 Cit. en Rojas, 2005).

A pesar de las diferentes significaciones en torno a lo que es ser académico, subsiste una imagen ideal del académico polifuncional y dedicado a contribuir en su rol social a las necesidades de la universidad como espacio de cultivo del conocimiento, su generación y comunicación involucrando las diferentes actividades académicas (docencia, investigación, gestión y extensión), aunque con recursos limitados (tiempo y financiamiento). A la vez, estas actividades conllevan un status diferenciado en el que la investigación recibe la mayor parte del prestigio (y las compensaciones económicas), lo cual promueve la competencia y una 'fragilidad reputacional' (Guzmán y Barnett, 2013a). En tal sentido, el status y prestigio son deseables, aunque transitorios, y llevan al académico y a la universidad a la que pertenece a una búsqueda permanente que los asegure. Las nociones de mercado, que influencian al mundo universitario y los juicios de valor sobre la productividad, hacen cada vez más difícil que los académicos puedan verse correspondiendo a esta imagen ideal de ser académico.

Se trata entonces de la construcción de identidades desde una macro complejidad - un sistema universitario mercantilizado y precario - que se plasma en una institución universitaria que promueve la polifuncionalidad y el multipropósito y, en última instancia, dan cuenta tanto de una fragilidad ontológica (complejidad en la naturaleza de ser académico) como reputacional (Guzmán-Valenzuela y Barnett, 2013a). A la vez, es un espacio que implica ciertas definiciones institucionales que, en algunos casos, pueden ser más o menos restrictiva dependiendo de las misiones y necesidades institucionales, las configuraciones disciplinares y las unidades académicas a las que pertenecen los académicos y que tienen particularidades históricas. Pero también involucra la posibilidad de desempeñar diferentes actividades con diferentes propósitos bajo un sistema de trabajo que otorga ciertos espacios para la libertad o el ejercicio de la agencia (Archer, 1995, 2003). Estas condiciones suponen tensiones que conviven en el discurso de los académicos sin ser resueltas. Al mismo tiempo, constituyen desafíos que los académicos enfrentan, en muchas ocasiones, como una oportunidad para potenciar microprácticas cotidianas en interacción con otros, que los llevan a alcanzar su realización y a crear oportunidades de satisfacción.

Este tipo de estudios, construidos desde las significaciones subjetivas de los actores involucrados, en este caso los académicos de una universidad compleja, permiten obtener información rica que puede ser de utilidad a la hora de implementar lineamientos institucionales estratégicos, así como reflexionar y establecer líneas de acción y políticas públicas en educación superior que respondan a las prioridades educativas nacionales en un mundo globalizado. Por último, para investigaciones y publicaciones a futuro, consideramos relevante abordar la perspectiva de género en detalle, la de académicos en situaciones contractuales precarias, así como proponer líneas de desarrollo académico que puedan ser implementadas en la universidad. 


\section{REFERENCIAS BIBLIOGRÁFICAS}

Archer, M. (1995). Realist social theory: the morphogenetic approach. Cambridge: Cambridge University Press.

Archer, M. (2003). Structure, Agency and the Internal Conversation. Cambridge: Cambridge University Press.

Barnett, R. (2011). Being a university. London: Routledge.

Barnett, R., \& DiNapoli, R. (2008). Changing identities in higher Education. Voicing Perspectives. Oxford: Routledge.

Becher, T. (2001). Tribus y territorios académicos. La indagación intelectual y las culturas de las disciplinas. Barcelona: Gedisa.

Bernasconi, A. (2006). Does the affiliation of universities to external organizations foster diversity in private Higher Education? Chile in comparative perspective. Higher Education, 52(2), 303-342.

Bernasconi, A. (2010). La apoteosis del investigador y la institucionalización de la profesión académica en Chile. Estudios sobre educación, 19, 139-163.

Berríos, P. (2007). Análisis sobre las profesoras universitarias. Calidad de la educación, 26, 39-53.

Berríos, P. (2008). Normas y percepciones sobre carrera académica en Chile. Calidad en la Educación, 28, 40-52.

Brunner, J. J. (1997). From State to Market Coordination: The Chilean Case. Higher Education Policy, 10(3-4), 225-237.

Clark, B. (1991). El sistema de Educación superior: Una visión comparativa de la organización académica. México: Editorial Nueva Imagen.

Clark, B. (1998). Creating Entrepreneurial Universities: Organizational Pathways of Transformation. Oxford: Pergamon/ IAU.

Clegg, S. (2008). Academic identities under threat? British Educational Research Journal, 34(3), 329-345.

Denzin, N., \& Lincoln, Y. (2008). The landscape of Qualitative Research (3rd ed.). London: Sage Publications.

Erickson, F. (1989). Métodos cualitativos de investigación sobre la enseñanza. En M. Wittrock (Comp.), La investigación en la enseñanza II. Métodos cualitativos y de observación (1a reimp., pp. 125-301). Madrid: Paidós.

Fanghanel, J (2012). Being an Academic. New York: Routledge.

Galaz, J. (1999). La experiencia universitaria y la profesión académica. Revista Electrónica de Investigación Educativa, 1(1). Recuperado de http://redie.uabc.mx/redie/article/view/4/8

Gil-Antón, M. (2000). Los académicos en los noventa: ¿actores, sujetos, espectadores o rehenes? Revista Electrónica de Investigación Educativa, 2(1).

Goetz, J., \& LeCompte, M. (1988). Etnografía y diseño cualitativo en investigación educativa. Madrid: Morata.

Grediaga, R. (2000). Profesión académica, disciplinas y organizaciones. Procesos de Socialización y sus efectos en las actividades y resultados de los académicos mexicanos. México D. F.: Asociación Nacional de Universidades e Instituciones de Educación Superior (ANUIES).

Grediaga, R. (2001). Retos y condiciones de desarrollo: la profesión académica en México en la última década. Revista Mexicana de Investigación Educativa, 6(11).

Grediaga, R. (2007). Tradiciones disciplinarias, prestigio, redes y recursos como elementos clave del proceso de comunicación del conocimiento. El caso mexicano. Sociológica, 22(65), 45-80. Recuperado de http://www.revistasociologica.com.mx/pdf/6503.pdf

Guba, E. (1989). Criterios de credibilidad en la investigación naturalista. En J. Gimeno y Pérez, A. (Eds.), La enseñanza: su teoría y su práctica (pp. 148-165). Madrid: Akal.

Guzmán-Valenzuela, C., \& Barnett, R. (2013a). Academic Fragilities in a marketized age: the case of Chile. British Journal of Educational Studies. 1-18. doi:10.1080/00071005.2013.776006 
Guzmán-Valenzuela, C., \& Barnett, R. (2013b). Marketing time: evolving timescapes in academia. Studies in Higher Education, 38(8), 1120-1134.

Guzmán-Valenzuela, C., \& DiNapoli. (2014). Competing narratives of time in the managerial university: the contradictions of fast time and slow time. En P. Gibbs, O. Ylijoki, C. GuzmánValenzuela \& R. Barnett (Eds.), The Flux of Time in the Contemporary University (pp. 154-167). Oxford: Routledge.

Henkel, M. (2005). Academic identity and autonomy in a changing policy. Environment Higher Education, 49, 155-176.

Lolas, F. (2008). Sobre académicos, academia y universidad. Calidad en la Educación, 28, 30-37.

McAlpine, L., \& Amundsen, C. (2011). Doctoral education: research based strategies for doctoral students, supervisors and administrators. Springer: London.

Marginson, S. (2010). 'Global Imagination', Model Two. Imagination: Three Models of Imagination in the Age of the Knowledge Economy (pp. 167-223). New York: Peter Lang.

Merriam, S. (1998). Qualitative Research and Case Studies Applications in Education. San Francisco: Jossey-Bass Publications.

Merino, L. (2004). El Sistema Universitario y la Vinculación Cultural con el Medio. Universidad y Sociedad, 20, Publicaciones Consejo Superior de Educación.

OECD. (2012). Education at a glance 2012. OECD. Recuperado de: http://www.oecd.org/edu/ EAG\%202012_e-book_EN_200912.pdf

Patton, MQ. (1999). Enhancing the quality and credibility of qualitative analysis. HSR: Health Services Research, 34(5), Part II, 1189-1208.

Rojas, G. (2005). Modelos Universitarios: Los rumbos alternativos de la universidad y la innovación. México: Fondo de la Cultura Económica.

Salord, S. (2001). Las trayectorias académicas: de la diversidad a la heterogeneidad. Revista Mexicana de Investigación Educativa, 6(11), 1-13.

Sánchez, A. (2002). Psicología Social Aplicada. Teoría, método y práctica. Madrid: Pearson Educación.

Santelices, B. (2011). Cumplimiento de las misiones universitarias por las Universidades Chilenas (Enseñanza, Creación de conocimiento y Transferencia Tecnológica). Estudios Sociales, 119, 93-130.

Simbürger, E. (2013). Moving through the city: visual discourses of upward social mobility in higher education advertisements on public transport in Santiago de Chile. Visual Studies, 28(1), 67-77.

Slaughter, S., \& Leslie, L. (1997). Academic Capitalism Politics, Policies, and the Entrepreneurial University. Baltimore: John Hopkins University Press.

Valimäa, J. (1998). Culture and identity in higher education research. Higher Education, 36, 119-138.

Van Maanen, J. (1982). Fieldwork on the beat. In J. Van Maanen, J. Dabbs \& R. Faulkner (Eds.), Varieties of Qualitative Research (pp. 103-115). Beverly Hills, CA: Sage Publications.

Vasilachis, I. (2006). La investigación cualitativa. En I. Vasilachis (Coord.), Estrategias de investigación cualitativa (pp. 23-54). Barcelona: Gedisa.

Ylijoki, O-H., \& Mäntylä, H. (2003). Conflicting Time Perspectives in Academic Work. Time \& Society, 12(1), 55-78. 